\title{
Plasmonic enhancement of SERS measured on molecules in carbon nanotubes
}

\author{
Niclas S. Mueller $\dagger^{\mathrm{a}}$ Sebastian Heeg, (D) $+\dagger^{\mathrm{b}}$ Patryk Kusch, ${ }^{a}$ \\ Etienne Gaufrès, ${ }^{c}$ Nathalie Y.-W. Tang, ${ }^{c}$ Uwe Hübner, ${ }^{d}$ Richard Martel, ${ }^{c}$ \\ Aravind Vijayaraghavan ${ }^{\mathrm{b}}$ and Stephanie Reich*a
}

Received 10th April 2017, Accepted 23rd May 2017

DOI: $10.1039 / c 7 f d 00127 d$

We isolated the plasmonic contribution to surface-enhanced Raman scattering (SERS) and found it to be much stronger than expected. Organic dyes encapsulated in single-walled carbon nanotubes are ideal probes for quantifying plasmonic enhancement in a Raman experiment. The molecules are chemically protected through the nanotube wall and spatially isolated from the metal, which prevents enhancement by chemical means and through surface roughness. The tubes carry molecules into SERS hotspots, thereby defining molecular position and making it accessible for structural characterization with atomic-force and electron microscopy. We measured a SERS enhancement factor of $10^{6}$ on $\alpha$-sexithiophene (6T) molecules in the gap of a plasmonic nanodimer. This is two orders of magnitude stronger than predicted by the electromagnetic enhancement theory $\left(10^{4}\right)$. We discuss various phenomena that may explain the discrepancy (including hybridization, static and dynamic charge transfer, surface roughness, uncertainties in molecular position and orientation), but found all of them lacking in enhancement for our probe system. We suggest that plasmonic enhancement in SERS is, in fact, much stronger than currently anticipated. We discuss novel approaches for treating SERS quantum mechanically that appear promising for predicting correct enhancement factors. Our findings have important consequences on the understanding of SERS as well as for designing and optimizing plasmonic substrates.

\section{Introduction}

Surface-enhanced Raman scattering (SERS) is the giant increase in the intensity of inelastic light scattering by molecules and nanosystems close to metallic

\footnotetext{
${ }^{a}$ Department of Physics, Freie Universität Berlin, 14195 Berlin, Germany. E-mail: sreich@zedat.fu-berlin.de ${ }^{b}$ School of Materials, The University of Manchester, Manchester M13 9PL, UK ${ }^{c}$ Regroupement québécois sur les matériaux de pointe, Département de Chimie, Université de Montréal, Montréal, QC H3C 3J7, Canada

${ }^{d}$ Leibniz Institute of Photonic Technology, o7745 Jena, Germany

$\uparrow$ These authors contributed equally.

† Present address: Photonics Laboratory, ETH Zürich, 8093 Zürich, Switzerland.
} 
nanostructures and rough metal surfaces. ${ }^{1-7}$ The Raman cross section of molecules in the vicinity of a metal is by many orders of magnitude stronger than for the same molecule in solution. ${ }^{8-11}$ SERS is a complex phenomenon with two sets of mechanisms that are known as chemical and plasmonic (electromagnetic, EM) enhancement. ${ }^{2,12,13}$ Plasmonic enhancement is induced by the electromagnetic near fields of the metal nanostructures. ${ }^{1,12}$ Metallic nanosystems possess collective excitations of their free electrons that are known as localized surface plasmons. They give rise to tightly focused and very intense near fields that enhance optical processes. ${ }^{\mathbf{1 , 3}, \mathbf{1 4}}$ Since both incoming and scattered radiation in a Raman experiment can be enhanced by the localized surface plasmon resonance (LSPR), plasmon excitation typically provides the strongest enhancement in a SERS process. ${ }^{8-11}$ Chemical enhancement refers to a SERS enhancement that arises from a chemical interaction between the metal and the Raman probe and sometimes also molecule-molecule interactions. ${ }^{\mathbf{1 3}}$ For example, the optical absorption may shift when molecules are placed on a metal surface, which may lead to an increase or decrease in the Raman response. ${ }^{15}$ Hybridized and charge transfer states of a metal and a molecule will induce additional optical excitations and introduce new resonances in the Raman or SERS cross section. ${ }^{\mathbf{1 6 - 1 9}}$ In the limit of very strong coupling between metal nanoparticles and molecules, the hybridization may even occur between the plasmonic excitations of the metal and the excitations of the Raman probe. ${ }^{18,20}$

Our understanding of SERS received a major boost when enhancement factors (EF, i.e., the ratio between the SERS and the Raman scattering cross section) were determined experimentally on individual, well-defined SERS hotspots. First measurements of $\mathrm{EF}$ for individual molecules were already performed on colloidal surfaces reporting hotspots with EFs reaching $10^{9}$ to $10^{10} \cdot{ }^{12-23}$ In recent years, individual nanostructures have been prepared via wet chemistry or nanoscale lithography, characterized microscopically, covered by molecules as Raman probes, and measured for their SERS enhancement. ${ }^{8-11}$ A great advantage of this approach is that the geometry of the nanoscale hotspot was accessible to scanning electron (SEM), transmission electron (TEM), and atomic force microscopy (AFM). This knowledge allowed for a precise modelling of the electromagnetic near fields of the plasmonic nanostructure. It turned out that the predictions of plasmonic enhancement within the EM enhancement theory underestimated the SERS enhancement by two to three orders of magnitude. ${ }^{8-11}$ This discrepancy is attributed to chemical enhancement and nanoscale roughness at the surface of the metal. The latter may introduce places of particularly strong field enhancement if the Raman probe is in close contact with a tiny spiky feature..$^{24,25}$ However, it was impossible to quantify these contributions independently.

Here we examine the plasmonic contribution to SERS measured on organic molecules encapsulated in carbon nanotubes (CNTs). Sexithiophene (6T) molecules were encapsulated into CNTs and placed into the gap of gold nanodimers. In our devices the CNTs act as carriers and cages for the molecules, they define the molecular position and orientation in the dimer cavity and make it accessible to SEM and AFM. We find an integrated SERS enhancement of $10^{4}$ to $10^{5}$, which corresponds to a maximum enhancement of $10^{6}$ to $10^{7}$ on an individual $6 \mathrm{~T}$ molecule. The modelled enhancement for the experimental geometry within the EM enhancement theory yielded EFs that were two orders of magnitude smaller than the experimentally obtained values. We extensively discuss additional 
mechanisms that may contribute to our SERS enhancement. The separation between $6 \mathrm{~T}$ molecules and the gold surface amounts at minimum to several nanometers, which prevents chemical interaction and renders atomic-scale roughness to be a negligible contribution within our system. The absence of chemical enhancement is further confirmed by Raman measurement on 6T molecules at the outer edge of a nanodimer. We thus propose that the plasmonic contribution to SERS is much stronger than anticipated to date. We discuss a recently developed description of SERS by a quantum mechanical treatment of the Raman effect and show that it is in good quantitative agreement with our experimental results.

\section{Experiments and simulation}

\section{Sample preparation and characterization}

We controllably deposited molecules in SERS hotspots, determined their orientation and position, and experimentally measured their enhancement. For this, 6T molecules were encapsulated in single-walled CNTs that act as molecular carriers. ${ }^{26,27}$ The SERS hotspots were produced by lithographically prepared gold nanodimers. ${ }^{28-31}$ We used dielectrophoresis to deposit the CNTs and 6T molecules in the dimer gap..$^{30-32}$ In the following we briefly describe the geometry and fabrication of the nanoplasmonic system, the filling of the CNTs with 6Ts, the deposition of the hybrids, and the characterization of the obtained devices. Details of the experiments will be published in a future study. ${ }^{33}$

The gold nanodimers were formed by two gold discs with $105 \mathrm{~nm}$ diameter and $75 \mathrm{~nm}$ height, Fig. 1(a). The gap between the discs $(20 \mathrm{~nm})$ forms a cavity, which is the place of the strongest electromagnetic near fields., ${ }^{3,1428}$ The nanodimers were fabricated in the centre between two electrodes, Fig. 1(b), that will be used for dielectrophoresis. ${ }^{30-32}$ The electrodes have a separation of $0.83 \mu \mathrm{m}$ and will be bridged by a nanotube after the deposition, see Fig. 1(c) and the description of the dielectrophoresis below. The dimer axes are oriented under an angle of $75^{\circ}$ with respect to the vector $\boldsymbol{s}$ connecting the two electrodes. This configuration decreases the angle between the dominant polarization direction of the electromagnetic
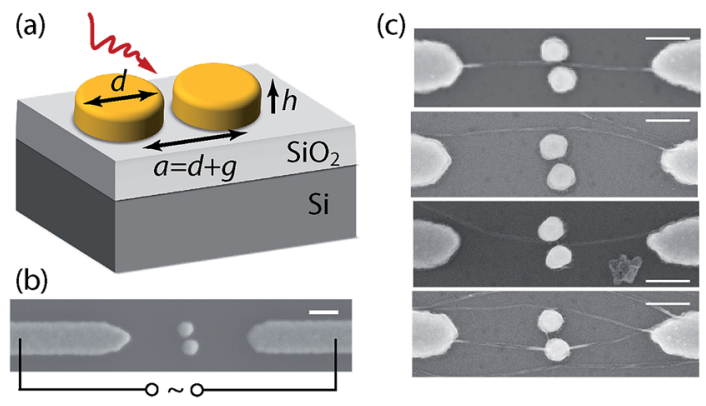

Fig. 1 (a) Geometry of a plasmonic nanodimer on a $\mathrm{SiO}_{2} / \mathrm{Si}$ substrate. The dimensions for our experiments were $d=105 \pm 5 \mathrm{~nm}, a=125 \pm 6 \mathrm{~nm}$, and $h=75 \pm 5 \mathrm{~nm}$. The $\mathrm{SiO}_{2}$ thickness was $290 \mathrm{~nm}$. (b) Exemplary nanodimer between the electrodes used for dielectrophoresis. (c) SEM images of 6TaCNT after deposition. The scale bars in (b) and (c) are $200 \mathrm{~nm}$. 
near field and the CNT axis. It increases the coupling between a CNT or an oriented molecule with the plasmonic excitation and thus the total SERS intensity. ${ }^{30,31}$

In the next step we aim at controllably depositing molecules in the dimer nanocavity. We use CNTs as nanocarriers by filling them with 6T. Such rod-like molecules pack neatly in the tight hollow space of the tubes. ${ }^{26}$ The $6 \mathrm{~T}$ form two double rows inside the CNT so that their principle axis is aligned with the axis of the tube. The encapsulated 6T species interacts with the CNT wall via a van der Waals interaction. Previous studies have demonstrated the absence of charge transfer and mixing of the electronic states of the two species. ${ }^{26,27,37} 6 \mathrm{~T}$ has a main Raman feature close to $1460 \mathrm{~cm}^{-1}$; it is well visible towards the background of the CNT vibrations at $1600 \mathrm{~cm}^{-1}$ (G mode) and $1350 \mathrm{~cm}^{-1}$ (D mode), see Fig. 2. We used plasma-torch single-walled CNTs (Raymor RN-020) with a mean diameter of $1.3 \mathrm{~nm}$ (diameter range $0.9-1.5 \mathrm{~nm}$ ); the filling procedure is described in ref. 26. For the dielectrophoresis a drop of 6T@CNT solution was placed on the gold electrodes applying an alternating electric field. ${ }^{30-32}$ It exerts a force onto the CNTs in the liquid, which aligns and deposits the 6T@CNT between the electrodes.

Due to their larger polarizability CNT bundles are deposited before individual tubes in DEP. ${ }^{34}$ We adjusted the experimental parameters so that only small bundles of 6T@CNT were deposited and no individual CNTs. In the SEM images of Fig. 1(c) we show a selection of representative devices with individual bundles inside the cavity, bundles outside the dimer, and electrodes with several 6T@CNT. For the SERS measurements we selected devices that contained only an individual6T@CNT bundle for a given pair of electrodes. The selection was based on AFM measurements. SEM images were taken after the Raman measurements to confirm the devise configuration. This prevented sample degradation and the immobilization of hydrocarbons on the imaged surfaces due to the electron beam used for imaging. We measured the diameter of the 6T@CNTs from height

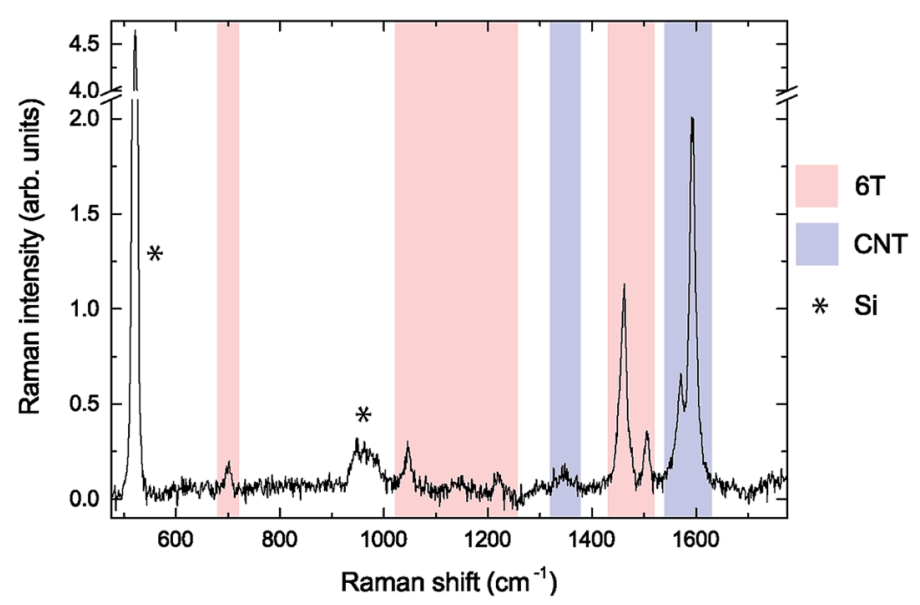

Fig. 2 Raman spectrum of a 6TaCNT hybrid on the $\mathrm{SiO}_{2} / \mathrm{Si}$ substrate. Raman features of 6T are marked in red and of CNTs in blue; features marked by an asterisk arise from the Si substrate. The excitation wavelength was $532 \mathrm{~nm}$; the incoming and scattered light were polarized along the 6TaCNT axis. 
profiles in AFM images. We found small bundles of CNTs with 8-10 nanotubes in a bundle. This implies 70-90 molecules per $10 \mathrm{~nm}$ deposited in each 6T@CNT segment. ${ }^{26}$ A key point of our approach is that the CNTs as molecular carriers define the molecular position and make it accessible to microscopy. We will later use this fact to build an accurate model of the $6 \mathrm{~T}$ molecules in the plasmonic dimer.

\section{SERS and Raman scattering}

For SERS and Raman experiments we selected 6T@CNTs with diameters of $5 \pm$ $2 \mathrm{~nm}$, as obtained by AFM. The constant diameter ensures that the number of excited 6T molecules remains approximately constant from one device to the next. Inelastic light scattering was excited using diode and tunable dye lasers. The laser was focused with an optical microscope $(100 \times$ objective, NA 0.9$)$ onto the plasmonic dimer. We maximized the SERS/Raman intensity by adjusting the $x, y$, and $z$ position of the nanodimer-6T@CNT device using a piezostage. Already a shift of the device position by $50 \mathrm{~nm}$ with respect to the laser spot resulted in a 15-20\% drop in overall scattering intensity. The elastically scattered light was suppressed by edge filters. The inelastically scattered light was analysed by a Raman spectrometer (Xplora, T64000, both Horiba) equipped with a CCD. We placed an analyser in front of the spectrometer so that we realized a scattering configuration were the incoming and scattered light are polarized parallel to each other. To choose the direction of the polarization with respect to the 6T@CNT and nanodimer axis we placed a $\lambda / 2$ wave plate in front of the microscope objective. By turning the $\lambda / 2$ plate we simultaneously rotated the polarization of the incoming and scattered light. ${ }^{35,36}$

Analysis of SERS enhancement factors. In the following we describe how we determined the SERS enhancement factors from the measured spectra. The total integrated enhancement factor in SERS is simply the ratio between the measured SERS intensity, $I_{\text {SERS }}$, and a reference intensity, $I_{\mathrm{R}}$,

$$
\mathrm{EF}_{\exp }=I_{\mathrm{SERS}} / I_{\mathrm{R}}
$$

The reference intensity is often measured on molecules in solution, because the signal from a molecular layer is too weak for Raman measurements, and/or a luminescence background prevents the recording of the Raman spectrum. The $6 \mathrm{~T}$ molecules in the CNTs have a strong intrinsic Raman signal that can be detected on a $\mathrm{SiO}_{2} / \mathrm{Si}$ substrate, see Fig. $2 .^{26,37}$ We therefore use a reference 6T@CNT to measure $I_{\mathrm{R}}$. To verify that the reference 6T@CNT and the 6T@CNTs used for SERS have a similar Raman response (same bundle thickness, filling fraction, etc.), we performed experiments with $532 \mathrm{~nm}$ excitation, i.e., outside the plasmonic resonance. ${ }^{33}$ The $6 \mathrm{~T}$ intensities of the devices used for determining $\mathrm{EF}_{\text {exp }}$ agreed to within $10 \%$ at $532 \mathrm{~nm}$ excitation.

A crucial point for the SERS and Raman experiments is the polarization of the incoming and scattered light. The nanodimer fixes the dominant near-field polarization direction to be parallel to the dimer axis. The dimer axis forms an angle, $\varphi$, with the CNT axis, see Fig. 1 . The Raman intensity $I_{\mathrm{R}}$ should be measured under the same polarization direction with respect to the 6T@CNT axis. This is technically impractical, because each 6T@CNT used for SERS has a slightly 
different $\varphi$. Also, the 6T@CNT are not visible under the optical microscope to verify the polarization direction in situ. Finally, the scattering intensity is extremely low, because $\varphi \approx 70^{\circ} \pm 10^{\circ}$ in our devices and the 6T and CNT Raman intensities drop with $\cos ^{4} \varphi$. We therefore measured $I_{\mathrm{R}}^{\max }$ when the incoming and scattered light are parallel to the axis of the reference 6T@CNT and determined the enhancement as

$$
\mathrm{EF}_{\exp }=\frac{I_{\mathrm{SERS}}}{I_{\mathrm{R}}^{\max } \cos ^{4} \varphi} .
$$

$\varphi$ was obtained from AFM and SEM images for each 6T@CNT. The $\cos ^{4} \varphi$ dependence of the Raman signal was verified on reference 6T@CNTs that were deposited outside the plasmonic nanodimer, see ref. 33 for details.

The experimental enhancement factor defined above ignores the fact that the SERS and the Raman experiment probe a vastly different number of molecules, because of the different effective scattering volume. While we found $\mathrm{EF}_{\exp }$ was best suited for a comparison to our simulations within the EM enhancement theory, SERS experiments normally report the enhancement of the Raman cross section $^{38}$

$$
\mathrm{EF}_{\mathrm{sc}}=\frac{I_{\mathrm{SERS}}}{N_{\mathrm{SERS}}} \frac{N_{\mathrm{R}}}{I_{\mathrm{R}}}
$$

$N_{\text {SERS }}\left(N_{\mathrm{R}}\right)$ are the number of molecules probed in the SERS (Raman) experiment. In our setup the molecular density is constant from one 6T@CNT to the next, implying $N_{\mathrm{R}} / N_{\text {SERS }}=V_{\mathrm{R}} / V_{\text {SERS }}$ with the scattering volumes of the Raman $V_{\mathrm{R}}$ and SERS $V_{\text {SERS }}$ experiment. Along the radial direction of the CNT bundle the scattering length is determined by the radius of the 6T@CNT, which is identical for SERS and Raman scattering. The scattering length along the 6T@CNT axis, however, is given by the effective width of the excitation spot. In the Raman experiment we measured the focal width, $w_{\mathrm{L}}=605 \mathrm{~nm}$, by scanning over a CNT and recording the scattering intensity as a function of position. The effective length in the SERS measurements depends on the extension of the plasmonic hotspot. We estimated $w_{\text {SERS }}=16 \mathrm{~nm}$ from finite-difference time-domain (FDTD) simulations, see below. We thus obtain

$$
\mathrm{EF}_{\mathrm{sc}}=\frac{I_{\mathrm{SERS}}}{I_{\mathrm{R}}^{\max } \cos ^{4} \varphi} \frac{w_{\mathrm{L}}}{w_{\mathrm{SERS}}}=\mathrm{EF}_{\mathrm{exp}} \cdot 38 .
$$

We note that using $w_{\text {SERS }}$ from FDTD simulations introduces a circular argument in our analysis. Our evaluation will, therefore, concentrate on $\mathrm{EF}_{\exp }$ and provide $\mathrm{EF}_{\mathrm{sc}}$ mainly for comparison with other studies of SERS enhancement.

\section{Simulations}

We simulated the electromagnetic near field of the gold nanodimer on the $\mathrm{SiO}_{2} / \mathrm{Si}$ substrate with FDTD techniques (Lumerical FDTD Solutions software package). The geometry of the nanodimer was determined from the AFM and SEM images of the individual gold structures. The reference for the electric field was obtained by simulating the system without the plasmonic nanostructure. This additional 
step accounts for the change in electric field due to the optical interference at the $\mathrm{SiO}_{2} / \mathrm{Si}$ substrate. ${ }^{39,40}$

The simulations were performed at the wavelength of the laser excitation (638 $\mathrm{nm})$ and the scattered photons $(703 \mathrm{~nm})$. By multiplying the separate enhancement factors at these two wavelengths we obtained a local enhancement of the scattering amplitude. To compare this result to the experimental enhancement factor, $\mathrm{EF}_{\text {exp }}$, we first projected the electromagnetic near field onto the transition dipole of the $6 \mathrm{~T}$ molecules and calculated a projected local enhancement factor. ${ }^{33}$ Molecular position and orientation were found from the AFM and SEM images as explained below. We then integrated the projected enhancement over the size of the laser spot along the $6 \mathrm{~T}$ line. The reference signal was obtained by integrating the intensities as found in the simulation of the substrate without the plasmonic nanostructure along the $6 \mathrm{~T}$ line. Finally, we divided the integrated enhancement by the reference signal to obtain an enhancement factor, $\mathrm{EF}_{\mathrm{em}}$, that can be compared to $\mathrm{EF}_{\text {exp }}$.

\section{Results and discussion}

\section{Position and orientation of the 6T@CNT in the dimer cavity}

A common problem when analysing SERS enhancement is the unknown position and orientation of the molecules that contribute to the signal. ${ }^{2,41}$ Since the electromagnetic near fields are so tightly confined, any change in molecular position will strongly affect the scattering intensity. The same holds for molecular orientation with respect to the near-field polarization. In our system the plasmonic nanodimers and the 6T@CNT may be imaged by SEM and AFM, which makes the entire SERS geometry - plasmonic nanostructure plus molecular position and orientation - accessible experimentally. Fig. 3 shows an example of how we

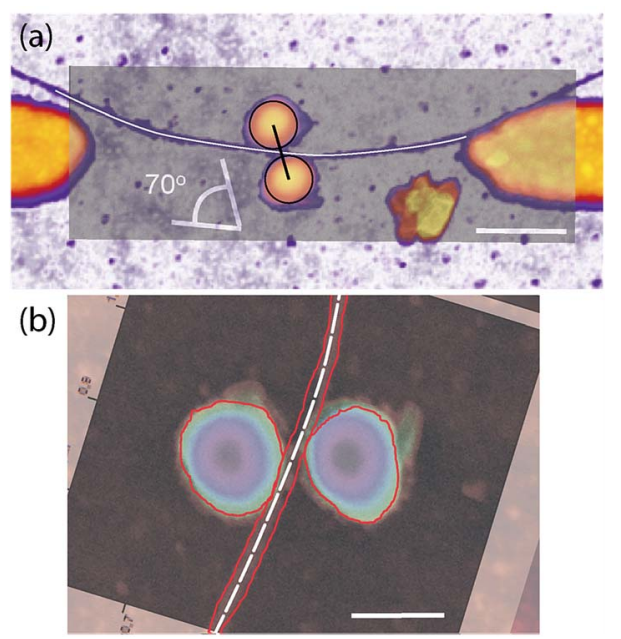

Fig. 3 Microscopy analysis of dimer geometry, 6T (CNT position and orientation. (a) Overview image of a 6T CCNT in a plasmonic cavity from interlaced AFM and SEM images. White line: traces the 6TaCNT, $\varphi=70^{\circ}$. (b) Zoomed image of the dimer. Red lines: AFM contour at a height of $3 \mathrm{~nm}$. White dashed line: central position of the 6T CCNT obtained in (a). Scale bars are $200 \mathrm{~nm}$ in (a) and $100 \mathrm{~nm}$ in (b), respectively. 
examined the experimental geometry of plasmonic nanodimer and 6T@CNT. The SEM images of the nanodimer were primarily used to access the disc diameter, $d$, the disc distance, $a$, and the gap size $g=a-d$, see Fig. 1 . Dimer height, $h$, and the three-dimensional shape were analysed from the AFM images. We paid particular attention to the position of the 6T@CNT in the dimer gap. We first extracted the 6T@CNT morphology and orientation from an overlay of an AFM and SEM image as shown in Fig. 3(a). A zoomed image, Fig. 3(b), was used to highlight an AFM contour (red line) at a constant height of $3 \mathrm{~nm}$, which corresponds to the centre of the CNT bundles with typical diameters of 5-7 nm. The 6T@CNT will appear much broader in the contour plot than its actual width, because of the finite diameter of the AFM tip. To finally determine the $6 \mathrm{~T}$ centre position we placed the bundle trace extracted from Fig. 3(a) into the contour of panel (b) (dashed white line). The minimum distance between the centre of the 6T@CNT and the gold surface is $\sim 7 \mathrm{~nm}$. We repeated this analysis for several 6T@CNTs. The bundles were preferentially deposited (almost) in the middle of the nanodimer gap. We only rarely found a bundle that was running over a disc or clearly attached to the gold surface. This systematic placement is characteristic for the dimers used here. Their rounded shape leads to a field line distribution that directs the 6T@CNT into the centre of the cavity during DEP, which will be published elsewhere.

The minimum distance of $7 \mathrm{~nm}$ between the centre of the 6T@CNT and the gold surface implies that the $6 \mathrm{~T}$ molecules are separated by at least $4 \mathrm{~nm}$ from the gold (we assumed a diameter of $5 \mathrm{~nm}$ ). In addition to the physical distance, at least one $\mathrm{sp}^{2} \mathrm{CNT}$ wall is present between the $6 \mathrm{~T}$ molecules and the metal. The distance and the shielding of molecule-metal interactions by the $\mathrm{sp}^{2}$ carbon sheet will be important when discussing chemical enhancement in SERS.

The $6 \mathrm{~T}$ transition dipole moments are oriented at an angle, $\varphi$, with respect to the nanodimer axis, $\varphi=70^{\circ}$ for the example 6T@CNT in Fig. 3. We previously showed that for the relatively large nanodimers used in our experiment, the SERS spectra originate from the projection of the light polarization onto the transition dipole of the molecule or the CNT. ${ }^{30}$ Strong gradients in the electric fields may induce scattering channels that are absent for standard Raman scattering. ${ }^{42,43}$ However, such effects are absent for the large gaps in our nanodimers $(20 \mathrm{~nm}) .{ }^{13}$ The large gap size also prevents distinct quantum mechanical effects such as tunnelling of electrons between the discs as well as rapid changes in the gap configuration due to mobile gold atoms. ${ }^{45}$

\section{Experimental SERS enhancement}

In previous studies we showed that the gold nanodimers of Fig. 1 may be coupled with two-dimensional graphene by transferring graphene monolayers onto the nanostructures. ${ }^{28,29}$ Using red laser excitation $(1.94 \mathrm{eV}$, around $640 \mathrm{~nm}$ ) reproducibly resulted in SERS enhancement, as we recently demonstrated, for 90 consecutive dimers in an analysis of local strain and doping of the graphene through the gold nanostructures. ${ }^{44}$ Interfacing the lithographic dimers with onedimensional CNTs was more challenging, ${ }^{30,31}$ but we succeeded by DEP deposition as also used for the 6T@CNT hybrids.

To measure the SERS intensity we excited the 6T@CNT with $638 \mathrm{~nm}$ laser excitation. In Fig. 4(a) we show a representative SERS spectrum taken on a 6T@CNT in a gold dimer in comparison to its reference spectrum. The SERS 


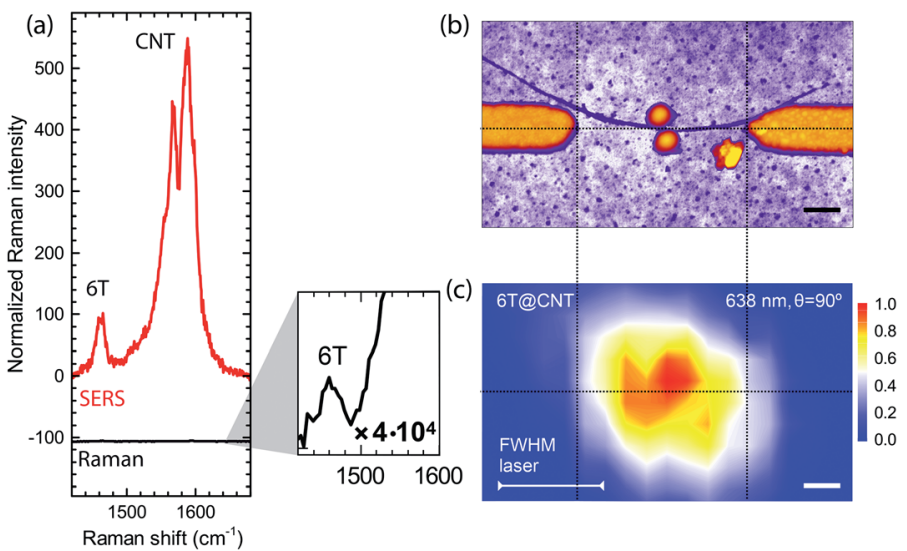

Fig. 4 (a) SERS spectrum of 6T and CNT peaks excited at $638 \mathrm{~nm}$ compared to a reference Raman spectrum (offset for clarity). The inset shows a zoomed image of the 6T peak of the reference. The polarization was parallel to the dimer in the SERS spectrum. For the reference we measured a 6 TACNT with light polarized along its axis and multiplied the spectrum by $\cos ^{4} 70^{\circ}$. (b) AFM image of a 6 T aCNT hybrid in the nanodimer cavity. (c) Map of the $6 \mathrm{~T}$ peak intensity as a function of $(x, y)$ position. The SERS intensity is strongly localized around the gap (compare to Fig. 8 for a corresponding map in the absence of SERS enhancement). The scale bar is $200 \mathrm{~nm}$.

intensity, $I_{\text {SERS }}$, exceeds the reference $I_{\mathrm{R}}$ by $\mathrm{EF}_{\exp }=8 \times 10^{4}$. We measured four other nanodimers where individual 6T@CNT of comparable diameter were positioned in the centre of the nanodimer cavity. ${ }^{33} \mathrm{EF}_{\text {exp }}$ varied between $3 \times 10^{4}$ and $9 \times 10^{5}$, which is a reasonable range given the variations in dimer shape, resonance frequency, and6T@CNT position. The enhancement of the scattering cross section for the 6T@CNT in Fig. 4 was $\mathrm{EF}_{\mathrm{sc}}=3 \times 10^{6} ; \mathrm{EF}_{\mathrm{sc}}$ varied between 1 $\times 10^{6}$ and $4 \times 10^{7}$ for the other $6 \mathrm{~T}$ hybrids.

Two additional experimental findings clearly demonstrate SERS enhancement in our system. First, the signal for $638 \mathrm{~nm}$ excitation, i.e., in resonance with the LSPR, was strongly localized around the nanodimer, Fig. 4(c). An analysis of the intensity distribution in Fig. 4(c) reveals a point-like hotspot that is folded with the laser focus in the experiment. ${ }^{28,30}$ We also found that the 6T@CNT SERS signal is fully polarized along the dimer axis. ${ }^{33}$ When the incoming and scattered light are polarized parallel to the nanodimer they couple to the strongest LSPR of the plasmonic system, which is in resonance with the chosen laser energy. Consequently, the intensity is strongest in this configuration and vanishes when turning the polarization perpendicular to the dimer axis. ${ }^{30,33}$ In particular, the SERS intensity is low when the light is polarized parallel to the 6T@CNT. In standard Raman scattering the intensity originates only from the projection of the electromagnetic field along the CNT axis. ${ }^{35,36}$ Having established the experimental SERS enhancement, we will now determine the plasmonic and chemical contribution to the enhancement factor.

\section{Simulations within EM enhancement theory}

The plasmonic contribution to SERS is modelled within the EM enhancement theory using the dimer geometry extracted from the AFM and SEM images. Fig. 5 

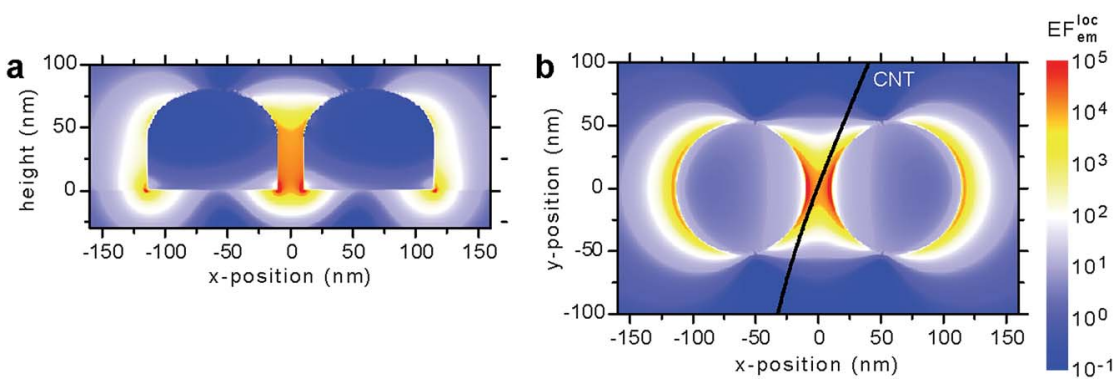

Fig. 5 Enhancement factors calculated within the EM enhancement theory using FDTD simulations. (a) Side view going through the inversion centre of the dimer. (b) Top view of the spatial distribution of the local enhancement factor. It was evaluated at $2.5 \mathrm{~nm}$ height corresponding to the mid point of the 6TaCNT. The black line indicates the position of the 6 TaCNT extracted from the microscopy images.

shows the local SERS enhancement factor calculated for an excitation at $638 \mathrm{~nm}$ and the 6T Raman frequency $1460 \mathrm{~cm}^{-1}$ (scattered radiation at $703 \mathrm{~nm}$ ). The simulation was evaluated at the centre height of the $5 \mathrm{~nm}$ thick6T@CNT bundle in Fig. 3 and 4(b). The maximum calculated local EF reached $1.4 \times 10^{5}$ compared to $\mathrm{EF}_{\mathrm{sc}}=3 \times 10^{6}$ observed experimentally. In contrast to individual 6T molecules, however, the molecules in the 6T@CNT hybrid cannot sample all positions in the $(x, y)$ plane of the nanocavity, but are confined to the place of the CNT bundle. We integrated the projected 6T EM enhancement along the line of the CNT and found the enhancement factor $\mathrm{EF}_{\mathrm{em}}=810$. This value is to be compared to the experimental $\mathrm{EF}_{\exp }=8 \times 10^{4}$ that is two orders of magnitude stronger. At first one may argue that the discrepancy results from small changes of the experimental $6 \mathrm{~T}$ arrangement and variations in the geometry of the nanodimer. We will now verify that the calculated EM enhancement for the encapsulated 6T molecules depends only weakly on the remaining uncertainties.

The position and orientation of the 6T@CNT line was determined from SEM and AFM images. The margin of error on the geometry is in the order of nm. We therefore systematically varied position and orientation of the 6T line, Fig. 6(a)(c), along which we integrate to obtain $\mathrm{EF}_{\mathrm{em}}$. The calculated $\mathrm{EF}_{\mathrm{em}}$ for the various configurations reached up to 1100, if the 6T@CNT was put in direct contact with the gold, Fig. 6(b), but overall the changes were quite small. We evaluated how strongly the shape of the nanodimer and the presence of the gold electrodes for dielectrophoresis affect the EM enhancement. The electromagnetic near field of the nanodimer and its projection onto the 6T line barely changed, Fig. 6(f), when using half-spherical gold discs instead of the cylinder plus ellipsoid, Fig. 6(e), or including the gold DEP electrodes in the simulations, Fig. 6(d). Since these simulations were computationally very expensive, we did not carry out the full calculation of $\mathrm{EF}_{\mathrm{em}}$, but conclude that the effects of discs shape and gold electrodes onto SERS enhancement are negligible.

Very strong and highly localized EM enhancement is expected if molecules are placed in the hotspot of tiny, atomic-scale bumps or protuberances of a nanoscale structure. ${ }^{24,25}$ Recently, mobile gold atoms were even shown to give rise to picocavities with huge SERS enhancement factors. ${ }^{45,46}$ To examine whether surface roughness may increase the calculated $\mathrm{EF}_{\mathrm{em}}$ and bring it closer to $\mathrm{EF}_{\exp }$, we 

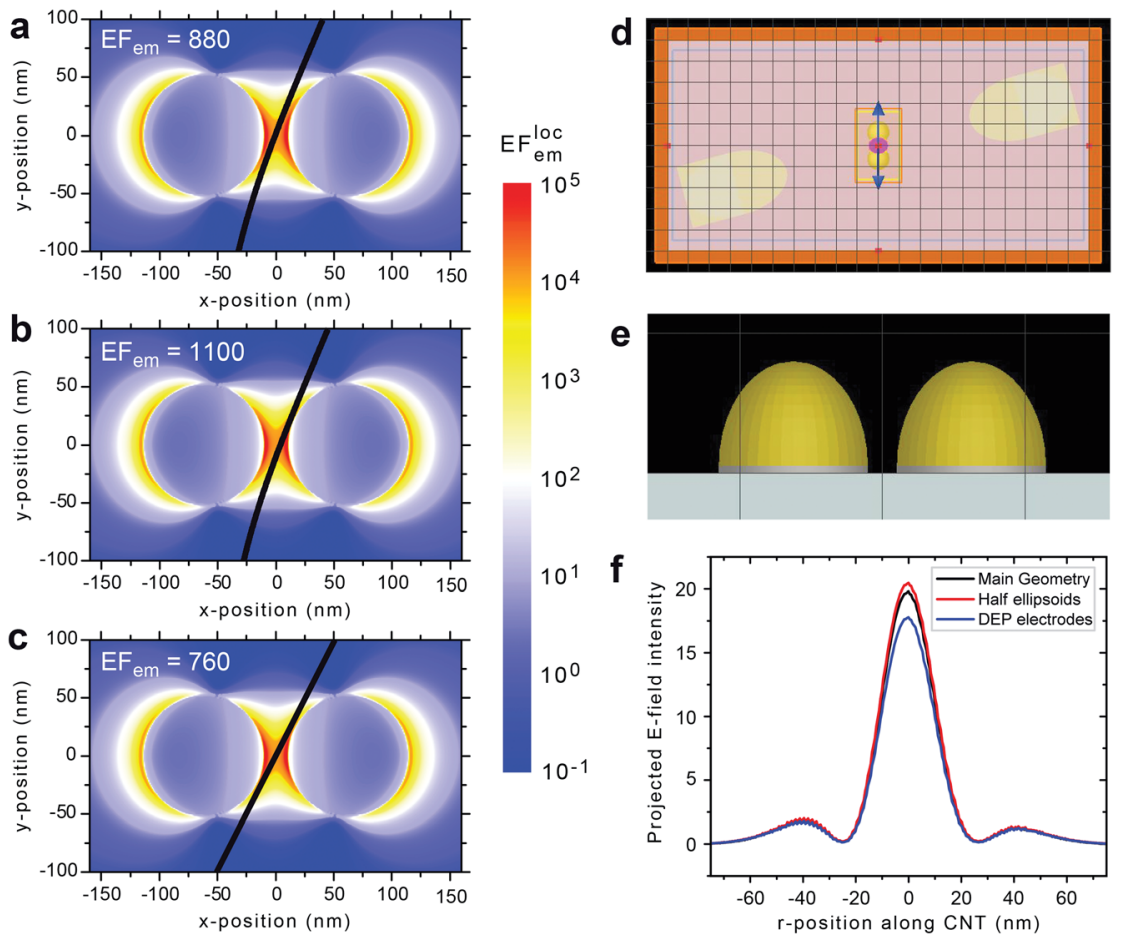

Fig. 6 Dependence of the EM enhancement on the device geometry. (a and b) Shift of the 6 TaCNT position with $\mathrm{EF}_{\mathrm{em}}$ given in the inset. (c) Rotation of the 6TaCNT for the minimum possible $\varphi$. (d) Simulation box containing the DEP electrodes in addition to the dimer. (e) Gold dimer with rounded, half-spherical shape. (f) Comparison between the original enhancement of the electric field intensity at $638 \mathrm{~nm}$ (black) and the geometries of (d) (blue) and (e) (red).

simulated a bump by attaching a gold nanoparticle to one of the discs as shown in Fig. 7. There is indeed a strong increase of the enhancement close to the nanoparticle. The maximum local SERS enhancement factor increased by a factor of 40

a
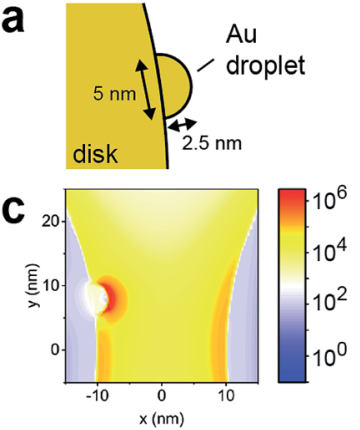

b

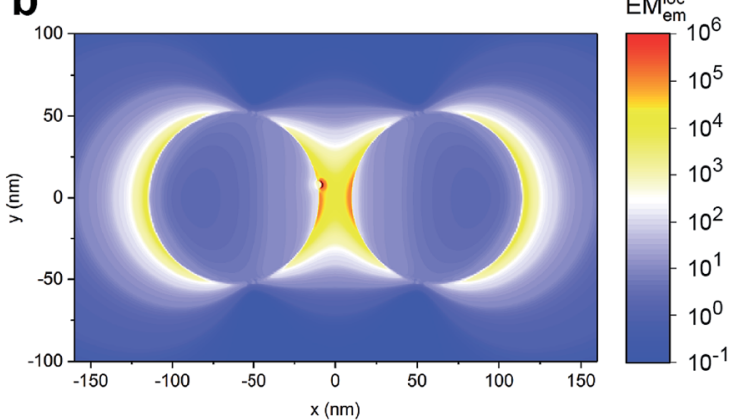

Fig. 7 Simulation of surface roughness. (a) Top view of the dimer geometry with a small protuberance in the form of a half-spherical particle with $2.5 \mathrm{~nm}$ radius. (b) Simulated EM enhancement. (c) Zoomed region of the nanoparticle. A $0.25 \times 0.25 \times 0.25 \mathrm{~nm}^{3}$ mesh was used in the FDTD simulations for the area shown in (c). 
compared to the smooth surface in Fig. 5. However, the hotspot induced by the local surface roughness extends to less than a $\mathrm{nm}$. The total enhancement factor, therefore, increases only by $28 \%\left[\mathrm{EF}_{\mathrm{em}}=1040\right.$, calculated from Fig. $\left.7(\mathrm{~b})\right]$. Surface roughness does not explain the strong SERS enhancement observed experimentally.

The simulations of the SERS enhancement within the EM enhancement theory are two to three orders of magnitude smaller than the experimental values. This result proved robust towards uncertainties in the experimental geometry, i.e., exact dimer shape, geometrical parameters, position and orientation of the 6T@CNT in the dimer gap. Because of the extension of the 6T@CNT hybrid and its distance to the gold surface, the effect of surface roughness is negligible and cannot explain the strong experimental SERS enhancement.

\section{Chemical enhancement}

Chemical enhancement summarizes a number of effects that arise from molecule-metal and molecule-molecule interactions., ${ }^{2,13,16,17}$ In general, chemical interaction provides less enhancement than the plasmonic mechanism except in special cases. ${ }^{16}$ The effect of the chemical mechanisms in SERS is often estimated to be in the order of $10^{2}$ to $10^{3}$, i.e., the order of magnitude that we observe for the difference between our measured SERS enhancement factors and the calculations of the EM enhancement theory.

We focus the following discussion on the interaction between the $6 \mathrm{~T}$ molecules and the metal. Molecule-molecule interaction is known to increase the scattering cross section of the 6T molecules in the 6T@CNT hybrids, because the molecules form an ordered aggregate. ${ }^{37}$ However, molecule-molecule interactions are irrelevant for our analysis, because we refer to the enhancement factors to Raman scattering intensities collected on 6T@CNT hybrids on the substrate. The same consideration holds for nanotube-molecule interactions. In previous studies some of us analysed the dependence of the $6 \mathrm{~T}$ intensity on the encapsulating CNT and found no correlation with the electronic character of the tube (semiconducting, metallic) and the intrinsic Raman resonances of the CNTs. ${ }^{26}$

We argue that the encapsulated 6T molecules experience no chemical enhancement, because they are too far away from the gold surface and are shielded by the CNT walls. Before considering possible ways of Raman enhancement through chemical interaction, we will present experimental evidence for the absence of chemical enhancement in our experiment. ${ }^{33} \mathrm{We}$ measured the Raman intensity as a function of position at $532 \mathrm{~nm}$ laser excitation. This wavelength is outside the resonance range of the LSPR. This was demonstrated for graphene by some of us in ref. 28; it was confirmed for the 6T@CNT by the polarization dependence of the scattering signal; the Raman

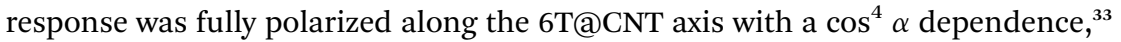
where $\alpha$ is the angle between the polarization of the incoming and scattered light with respect to the 6T@CNT axis..$^{26,35,36}$ Note that with plasmonic enhancement the signal was at maximum for light polarization parallel to the dimer. ${ }^{33}$

In the absence of the plasmonic contribution, a change in the Raman cross section is due to chemical enhancement. We mapped the intensity distribution of the $6 \mathrm{~T}$ and CNT signal, Fig. 8. Both signals varied by less than $40 \%$ close to the gold; in particular, there was no concentration of the intensity at the dimer 
structure as found under plasmonic enhancement in Fig. 4(c). We also examined one of the few6T@CNTs that was deposited on top of a gold nanodisc, Fig. 9(a), to detect an enhancement that was not plasmonic in origin. The Raman signal, Fig. 9(b), shows the typical signatures of the 6T and CNT Raman modes with $532 \mathrm{~nm}$ excitation. When switching to red excitation $(638 \mathrm{~nm})$ no signal could be detected on this particular 6T@CNT hybrid. The absence of plasmonic enhancement in the 6T@CNT configuration of Fig. 9 is explained by the distribution of the SERS enhancement around a nanodimer. Nanoscale mapping of the SERS intensity of the plasmonic dimers using a dual Raman-Rayleigh scattering scanning near-field optical microscope shows that the SERS enhancement vanishes on top of the nanodiscs. ${ }^{47}$ The presence of chemical enhancement at $638 \mathrm{~nm}$ should still lead to a strong and detectable 6T signal, which is clearly not the case in the measurements of Fig. 9.

"Chemical enhancement" is a broad term that refers to different effects in the interaction between molecules and metal, see e.g. ref. 13 for a review. Briefly, the Raman polarizability changes with a change in the configuration of the molecular ground state due to the metal. Le Ru and co-workers showed recently that this is a common phenomenon for molecules that were in direct contact with silver nanoparticles. ${ }^{15}$ A molecular Raman resonance may also be interpreted as additional SERS enhancement when the reference intensity, $I_{\mathrm{R}}$, is measured on a different molecular species or under very different conditions (this effect is excluded by our choice of reference). Finally, the close proximity between molecule and metal may also result in charge transfer resonances of the Raman effect. We now discuss the possibility of changes in the molecular configuration and of charge transfer excitations in the nanodimer-6T@CNT system.

A change in the ground state configuration may originate from the formation of a chemical bond, hybridized states, and static charge transfer. ${ }^{13}$ We exclude the

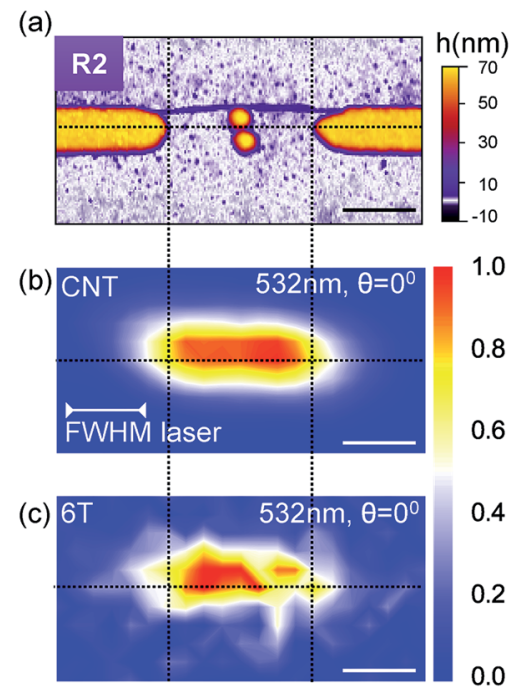

Fig. 8 (a) AFM image of a 6TaCNT molecule touching the outer edge of a nanodimer. Raman intensity maps of the (b) CNT G mode, and (c) 6T mode. The intensities are evenly distributed over the length of the hybrid. Incoming $(532 \mathrm{~nm})$ and scattered light are polarized along the 6T@CNT axis. The scale bars are $400 \mathrm{~nm}$. 
(a)

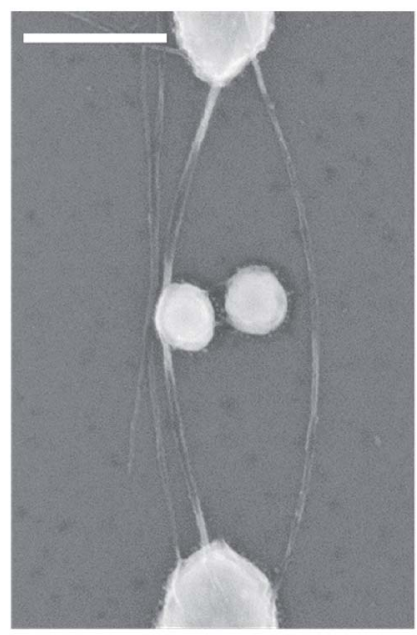

(b)

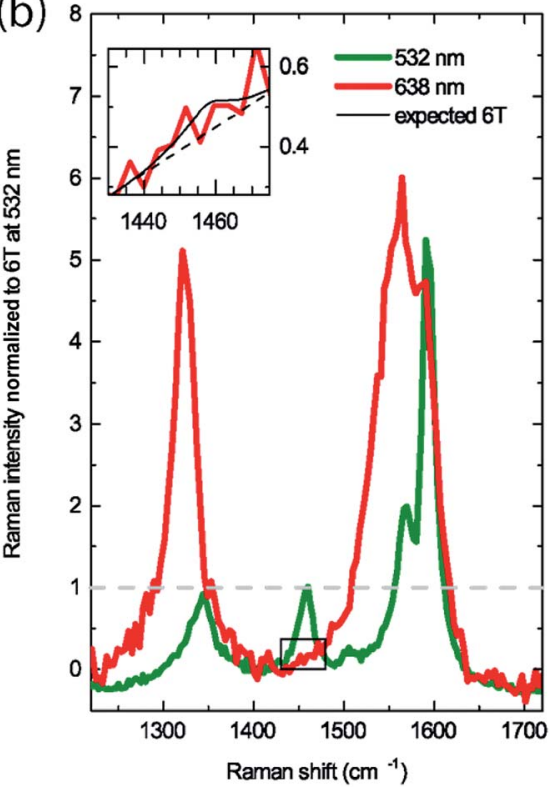

Fig. 9 (a) SEM image of a 6TaCNT touching the outer edge of a gold dimer plus other hybrids that are not in touch with the dimer. The scale bar is $200 \mathrm{~nm}$. (b) Raman spectra of the device in (a) collected at $638 \mathrm{~nm}$ (red) and $532 \mathrm{~nm}$ (green) with light polarized along the 6 TaCNT. The inset shows the expected 6T Raman intensity at $638 \mathrm{~nm}$ (black line) based on the spectrum measured for green excitation together with the recorded spectrum (red). The intensity was adjusted to account for substrate interferences, the pre-resonance condition of the $6 \mathrm{~T}$ molecules at $532 \mathrm{~nm}$, and the sensitivity of the setup. Clearly, no enhancement by a non-plasmonic mechanism is present.

formation of chemical bonds between the 6T and the gold and other effects that require a direct overlap of the molecular and metal wavefunction like hybridization and a change in conformation, because the molecules are several nm away from the gold and protected by the $\mathrm{sp}^{2}$ carbon sheet. Charge transfer to the $6 \mathrm{~T}$ molecules does not occur, because the CNTs shield their interior against charges. This was shown by Kavan and co-workers, ${ }^{48,49}$ who charged 6T@CNT and $\mathrm{C}_{60} @ \mathrm{CNT}$ in an electrochemical cell and performed in situ Raman experiments. While the CNTs were clearly doped, no anion or cation formation was observed for the encapsulated species. A static charge transfer shifts the frequencies and intensities of the $6 \mathrm{~T}$ Raman modes, ${ }^{50}$ which we did not observe experimentally. Both the 6T and CNT Raman lines were at their intrinsic positions. ${ }^{33}$ Transient effects that change the Raman response close to a metal are charge transfer resonances. They occur if an electron in the metal is promoted by the excitation laser into the excited state of a molecule yielding an adsorbate anion in the excited state. ${ }^{16}$ Charge transfer transitions require close proximity between molecule and metal, which does not exist in our system. The phenomena giving rise to chemical enhancement require a very small distance between molecule and metal, whereas the $6 \mathrm{~T}$ molecules are at least several $\mathrm{nm}$ away from the gold. 


\section{Discussion of SERS enhancement in 6T@CNT}

We briefly summarize the experimental findings, simulations, and considerations collected so far. We deposited 6T molecules in the gap of a gold nanodimer. The obtained devices were imaged by AFM and SEM, which gave us access to the real geometries of the dimer as well as the position and orientation of the 6T molecules. We recorded SERS spectra of 6T molecules in the dimer gap and reference spectra from 6T@CNT hybrids lying outside the dimer on the $\mathrm{SiO}_{2} / \mathrm{Si}$ substrate. From these experiments we obtained an integrated SERS enhancement factor $\mathrm{EF}_{\text {exp }}=10^{4}$ to $10^{5}$, and an enhancement of the Raman cross section $\mathrm{EF}_{\mathrm{cs}}=10^{6}$ to $10^{7}$. We simulated the plasmonic enhancement using the EM enhancement theory. The experimental $\mathrm{EF}_{\exp }$ exceeds the calculated $\mathrm{EF}_{\mathrm{em}}$ by a factor $10^{2}$.

We examined various mechanisms that may provide additional enhancement for the SERS measurements: (i) geometrical details of the nanostructure including changes in shape, nanometre roughness, and the presence of the electrodes for dielectrophoresis; (ii) remaining uncertainties in the position and orientation of the 6T@CNT; (iii) chemical enhancement from an experimental point of view; (iv) change in the 6T ground state configuration; and (v) charge transfer resonances between the $6 \mathrm{~T}$ molecules and the gold. We found that none of the mechanisms provides an orders of magnitude additional enhancement.

There are more exotic mechanisms that are found in SERS experiments, but are excluded in our devices. For example, field gradients may provide a separate enhancement channel and activate modes in SERS that are not normally Raman active. ${ }^{13,42,43,51}$ The large size of the nanodimer gap ensures that field gradients are sufficiently small. We estimated the second, gradient-related term in the multipole polarizability expansion of the dipole moment and found it to be at least an order of magnitude weaker than the first term that is related to the magnitude of the electric field.$^{13}$ Another idea is that the CNTs provide additional enhancement by inducing an optical near field in their interior. Gaufrès et al. ${ }^{37}$ studied this effect for the6T@CNT hybrids and found that the enhancement is on the order of one, i.e., the Raman signal gets neither enhanced nor reduced. We are happy to discuss other suggestions that are compatible with our device. The strongest constraints arise from the well-established geometry of our system and the fact that neither the 6T molecules nor the 6T@CNT hybrids are in direct contact with the metal. Also, any additional enhancement effect needs to be strong enough to account for two orders of magnitude difference in total scattering intensity.

Our understanding of the experimental results is that we isolated and measured the plasmonic contribution to SERS. The plasmonic enhancement in SERS is much stronger than anticipated to date. This is an excellent result for the nanoplasmonic and the SERS community, because single-molecule SERS and other techniques requiring superior enhancement are much easier to implement than expected up to now. Plasmonic effects can also be modelled and optimized accurately, which greatly helps in rationally tailoring SERS systems.

\section{Novel approaches for describing plasmonic enhancement in SERS}

At the end of our contribution we want to propose a solution for the discrepancy between the experimental and calculated strength of the plasmonic contribution to SERS. ${ }^{33}$ It is based on novel ways for describing plasmonic enhancement in SERS. Mueller et al. ${ }^{52}$ recently suggested treating SERS within the microscopic 
description of the Raman effect in solids and essentially understanding it as higher-order Raman scattering (HORa). Roelli et al. ${ }^{53}$ analysed SERS in analogy to quantum optomechanical systems, similar models were pursued by Aizpurua and collaborators. ${ }^{45,54}$ Both approaches arrived at similar expressions for the SERS cross section (we present the HORa formalism that includes more interaction pathways $)^{50}$

$$
\begin{aligned}
& \operatorname{EF}_{\mathrm{HORa}}\left(\omega_{\mathrm{L}}\right)= \\
& \left|1+\frac{M_{1} M_{2}}{\left(\hbar \omega_{\mathrm{L}}-E_{\mathrm{pl}}+\mathrm{i} \gamma\right)\left(\hbar \omega_{\mathrm{s}}-E_{\mathrm{pl}}+\mathrm{i} \gamma\right)}+\frac{M_{1}}{\left(\hbar \omega_{\mathrm{L}}-E_{\mathrm{pl}}+\mathrm{i} \gamma\right)}+\frac{M_{2}}{\left(\hbar \omega_{\mathrm{s}}-E_{\mathrm{pl}}+\mathrm{i} \gamma\right)}\right|^{2},
\end{aligned}
$$

where $\hbar \omega_{\mathrm{L}}$ is the energy of the laser, $\hbar \omega_{\mathrm{s}}$ the energy of the scattered light, $E_{\mathrm{pl}}$ the eigenenergy of the plasmon, and $\gamma$ the line width of the plasmonic resonance. The combined matrix elements $M_{1}$ and $M_{2}$ describe the coupling between photons, plasmons, and the electronic excitations of the Raman probe. The descriptions of the SERS mechanism within perturbation theory predict the appearance of plasmon-induced resonances (similar was introduced for charge transfer resonances previously). It is conceptually different from the antenna-like mechanism underlying the EM enhancement theory.

The challenge in calculating $\mathrm{EF}_{\mathrm{HORa}}$ lies in calculating the matrix elements $M_{1}$ and $M_{2}$. We estimated the enhancement predicted within the HORa approach using the results of a quasi-static approximation of a dimer that consisted of two nanospheres with overall $<50 \mathrm{~nm}$ lateral dimension, mapping the results back onto the realistic geometry; the details of our approach will be published elsewhere. ${ }^{33}$ We obtained $\mathrm{EF}_{\mathrm{HORa}}=1.4 \times 10^{5}$ which compares favourably with the experimental $\mathrm{EF}_{\exp }=8 \times 10^{4}$. The predictions of the novel quantum mechanical theories of SERS appear to be promising. They open an interesting route to pursue in the future modelling of SERS. Accurate, quantitative SERS using these theories requires further work on calculating the combined matrix elements in eqn (1). ${ }^{50-52}$ It will give more insight into the fundamentals of plasmonic enhancement in SERS.

\section{Conclusions}

We placed organic molecules in a hotspot for SERS by depositing 6T filled CNTs in a gold nanodimer. This system gives a unique degree of control over the experimental device geometry, because molecular position, orientation, and the geometry of the plasmonic structure were determined by SEM and AFM. We observed strong and reproducible SERS enhancement when exciting in resonance with the LSPR of the nanodimer. The integrated (total) enhancement factors were two orders of magnitude stronger than predicted within the EM enhancement theory for the experimental device geometry. We showed that for our system no other enhancement mechanism provides an increase in the total scattering intensity by a factor of 100 . We propose that the plasmonic enhancement in SERS is intrinsically much stronger then assumed to date. Recent proposals for treating SERS as a quantum mechanical scattering process including plasmon excitation 
were examined. They are very promising for correctly predicting the plasmonic enhancement to SERS.

\section{Acknowledgements}

We thank K. Pippardt, B. Steinbach, M. Ziegler, and D. Franke for their technical support in the microfabrication of the DEP/plasmonic nanostructures, and A. Oikonomou for advice in dielectrophoretic deposition. This work was supported by the German Research Foundation (DFG via SFB 658, subprojects A6) and the Focus Area NanoScale of the Freie Universität Berlin. A. V. and S. H. acknowledge funding from the Engineering and Physical Sciences Research Council (EPSRC) grant EP/K016946/1. N. S. M. thanks Deutsche Telekom Stiftung for financial support. R. M. acknowledges financial support from the Institut de l'Énergie Trottier, Natural Sciences and Engineering Research Council of Canada (NSERC) and the Fonds de Recherche du Québec-Nature et Technologie (FRQNT).

\section{References}

1 L. Novotny and B. Hecht, Principles of Nano-Optics, Cambridge, 2012.

2 E. Le Ru and P. Etchegoin, Principles of Surface-Enhanced Raman Spectroscopy and related plasmonic effects, Elsevier, New York, 2008.

3 M. S. Tame, K. R. McEnery, S. K. Özdemir, J. Lee, S. A. Maier and M. S. Kim, Nat. Phys., 2013, 9, 329.

4 A. Murray and W. L. Barnes, Adv. Mater., 2007, 19, 3771.

5 D. L. Jeanmaire and R. P. Van Duyne, J. Electroanal. Chem. Interfacial Electrochem., 1977, 84, 1.

6 B. Sharma, R. R. Frontiera, A.-I. Henry, E. Ringe and R. P. Van Duyne, Mater. Today, 2012, 15, 16.

7 R. Zhang, Y. Zhang, Z. C. Dong, et al., Nature, 2013, 498, 82.

8 D.-K. Lim, K.-S. Jeon, J.-H. Hwang, H. Kim, S. Kwon, Y. D. Suh and J.-M. Nam, Nat. Nanotechnol., 2011, 6, 452.

9 K. L. Wustholz, A.-I. Henry, J. M. McMahon, R. G. Freeman, N. Valley, M. E. Piotti, M. M. Natan, G. C. Schatz and R. P. van Duyne, J. Am. Chem. Soc., 2010, 132, 10903.

10 W. Zhu and K. B. Crozier, Nat. Commun., 2014, 5, 5228.

11 D. Talaga, M. Comesana-Hermo, S. Ravaine, R. A. L. Vallee and S. Bonhommeau, J. Opt., 2015, 17, 114006.

12 M. Moskovits, Phys. Chem. Chem. Phys., 2013, 15, 5301.

13 L. Jensen, C. M. Aikens and G. C. Schatz, Chem. Soc. Rev., 2008, 37, 1061.

14 E. Prodan, C. Radloff, N. J. Halas and P. Nordlander, Science, 2003, 302, 5644.

15 B. L. Darby, B. Auguie, M. Meyer, A. E. Pantoja and E. C. Le Ru, Nat. Photonics, 2016, 10, 40 .

16 M. Osawa, N. Matsuda, K. Yoshii and I. Uchida, J. Phys. Chem., 1994, 98, 12702.

17 S. M. Morton and L. Jensen, J. Am. Chem. Soc., 2009, 131, 4090.

18 R. Chikkaraddy, B. de Nijs, F. Benz, S. J. Barrow, O. A. Scherman, E. Rosta, A. Demetriadou, P. Fox, O. Hess and J. J. Baumberg, Nature, 2016, 535, 127.

19 S. Huang, X. Ling, L. Liang, Y. Song, W. Fang, J. Zhang, J. Kong, V. Meunier and M. S. Dresselhaus, Nano Lett., 2015, 15, 2892. 
20 N. T. Fofang, T.-H. Park, O. Neumann, N. I. Mirin, P. Nordlander and N. J. Halas, Nano Lett., 2008, 8, 3481.

21 Y. Fang, N.-H. Seong and D. D. Dlott, Science, 2008, 321, 388.

22 T. A. Laurence, G. B. Braun, N. O. Reich and M. Moskovits, Nano Lett., 2012, 12, 2912.

23 A. D. McFarland, M. A. Young, J. A. Dieringer and R. P. van Duyne, J. Phys. Chem. B, 2005, 109, 11279.

24 A. Trügler, J.-C. Tinguely, G. Jakopic, U. Hohenester, J. R. Krenn and A. Hohenau, Phys. Rev. B: Condens. Matter Mater. Phys., 2014, 89, 165409.

25 G. Macias, M. Alba, L. F. Marsala and A. Mihi, J. Mater. Chem. C, 2016, 4, 3970.

26 E. Gaufrès, N. Y. Tang, F. Lapointe, J. Cabana, M. A. Nadon, N. Cottenye, F. Raymond, T. Szkopek and R. Martel, Nat. Photonics, 2013, 8, 72.

27 M. A. Loi, J. Gao, F. Cordella, P. Blondeau, E. Menna, B. Bártová, C. Hébert, S. Lazar, G. A. Botton, M. Milko, et al., Adv. Mater., 2010, 22, 1635.

28 S. Heeg, R. Fernandez-Garcia, A. Oikonomou, et al., Nano Lett., 2013, 13, 301.

29 S. Heeg, A. Oikonomou, R. Fernandez-Garcia, et al., Phys. Status Solidi RRL, 2013, 7, 1067.

30 S. Heeg, A. Oikonomou, R. Fernandez-Garcia, et al., Nano Lett., 2014, 14, 1762.

31 S. Heeg, N. Clark, A. Oikonomou, A. Vijayaraghavan and S. Reich, Phys. Status Solidi RRL, 2014, 8, 785.

32 A. Vijayaraghavan, S. Blatt, D. Weissenberger, M. Oron-Carl, F. Hennrich, D. Gerthsen, H. Hahn and R. Krupke, Nano Lett., 2007, 7, 1556.

33 S. Heeg, N. S. Mueller, U. Hübner, P. Kusch, E. Gaufrès, N. Y.-W. Tang, R. Martel, A. Vijayaraghavan and S. Reich, Nanoscale, 2017, unpublished.

34 R. Krupke, F. Hennrich, H. B. Weber, M. M. Kappes and H. von Löhneysen, Nano Lett., 2003, 3, 1019.

35 G. S. Duesberg, I. Loa, M. Burghard, K. Syassen and S. Roth, Phys. Rev. Lett., 2000, 85, 5436.

36 S. Reich, C. Thomsen, G. S. Duesberg and S. Roth, Phys. Rev. B: Condens. Matter Mater. Phys., 2001, 63, 041401.

37 E. Gaufrès, N. Y-W Tang, A. Favron, C. Allard, F. Lapointe, V. Jourdain, S. Tahir, C.-N. Brosseau, R. Leonelli and R. Martel, ACS Nano, 2016, 10, 10220.

38 E. C. Le Ru and P. G. Etchegoin, MRS Bull., 2013, 38, 631.

39 R. Narula, R. Panknin and S. Reich, Phys. Rev. B: Condens. Matter Mater. Phys., 2010, 82, 045418.

40 P. Klar, E. Lidorikis, A. Eckmann, I. A. Verzhbitskiy, A. C. Ferrari and C. Casiraghi, Phys. Rev. B: Condens. Matter Mater. Phys., 2013, 87, 205435.

41 E. C. Le Ru and P. Etchegoin, Annu. Rev. Phys. Chem., 2012, 63, 65.

42 E. J. Ayars, H. D. Hallen and C. L. Jahncke, Phys. Rev. Lett., 2000, 85, 4180.

43 A. Alabastri, X. Yang, A. Manjavacas, H. O. Everitt and P. Nordlander, ACS Nano, 2016, 10, 4835.

44 N. S. Mueller, S. Heeg, M. Peña Alvarez, P. Kusch, S. Wasserroth, N. Clark, F. Schedin, J. Parthenios, K. Papagelis, C. Galiotis, M. Kalbáč, A. Vijayaraghavan, U. Huebner, R. Gorbachev, O. Frank, and S. Reich, 2017, arXiv:1703.09592.

45 S. Trautmann, J. Aizpurua, I. Götz, et al., Nanoscale, 2017, 9, 391.

46 F. Benz, M. K. Schmidt, A. Dreismann, et al., Science, 2016, 354, 726. 
47 P. Kusch, S. Mastel, N. S. Mueller, N. Morquillas, S. Heeg, R. Gorbachev, F. Schedin, U. Huebner, J. I. Pascual, S. Reich and R. Hillenbrand, Nano Lett., 2017, 17, 2667.

48 M. Kalbac, L. Kavan, S. Gorantla, T. Gemming and L. Dunsch, Chem.-Eur. J., 2010, 16, 11753.

49 L. Kavan, L. Dunsch, H. Kataura, A. Oshiyama, M. Otani and S. Okada, J. Phys. Chem. B, 2003, 107, 7666.

50 N. Yokonuma, Y. Furukawa, M. Tasumi, M. Kuroda and J. Nakayama, Chem. Phys. Lett., 1996, 255, 431.

51 C. M. Aikens, L. R. Madison and G. C. Schatz, Nat. Photonics, 2013, 7, 508.

52 N. S. Mueller, S. Heeg and S. Reich, Phys. Rev. A, 2016, 94, 023813.

53 P. Roelli, C. Galland, N. Piro and T. J. Kippenberg, Nat. Nanotechnol., 2015, 11, 164.

54 M. K. Schmidt, R. Esteban, A. Gonzalez-Tudela, et al., ACS Nano, 2016, 10, 6291. 